\title{
Ultra-thin Rigid diagnostic and therapeutic arthroscopy during arthrocentesis: Development and preliminary clinical findings
}

\author{
Seong-Yong Moon ${ }^{1}$ and Hoon Chung ${ }^{2^{*}}$
}

\begin{abstract}
Arthroscopy is useful to detect early changes in the temporomandibular joint (TMJ). Despite great advances in arthroscopy, many arthroscopic surgeries have now been replaced by arthrocentesis. We propose a simple diagnostic and therapeutic method having operative rigid ultra-thin arthroscopy with 16 gauge needle size combined with arthrocentesis.
\end{abstract}

Keywords: Arthroscopy; Arthocentesis; TMD; Closed lock; Anterior disc displacement without reduction; Habitual dislocation

\section{Background}

Arthroscopy of the temporomandibular joint (TMJ) was first reported by Ohnishi [1]. Arthroscopy is useful to detect early changes of intra-articular space in the temporomandibular joint (TMJ) that can not be detected with magnetic resonance imaging (MRI) and computed tomography (CT) [2].

Arthrocentesis is regarded as being less invasive than arthroscopic lysis and lavage. In addition, the effectiveness of arthrocentesis has been shown to be clinically acceptable [3] and not different from that of arthroscopic surgery. However, conventional TMJ arthrocentesis does not provide any information in joint pathosis [4]. Operative arthroscopy provides benefits over arthrocentesis because of the ability to visualize the joint for diagnostic purposes and perform surgical maneuvers and it offers a good success rate [5]. Recently fiber ultra-thin arthroscopy is developed which is useful and valuable in examination of the pathologic TMJ, however the resolution of the view is unclear [6].

In this report, we propose a simple diagnostic and therapeutic method of operative ultra-thin rigid arthroscopy combination with arthrocentesis through the Chung's needle (16 gauge needle size).

\footnotetext{
*Correspondence: choontmj@chol.com

${ }^{2}$ Hoon Chung Dental Clinic, \#2305, Jangkyo BLDG.,1 Jangkyo-Dong, Seoul, Jung-Gu Zip Code: 110-760, Republic of Korea

Full list of author information is available at the end of the article
}

\section{Chung's needle and Arthroscopy}

1) Chung's needle (Fig. 1)

Chung's needle has $66.20 \mathrm{~mm}$ of total length, $43.50 \mathrm{~mm}$ of available length, $1.65 \mathrm{~mm}$ of external diameter, and $8.30 \mathrm{~mm}$ of irrigation port. Rigid arthoscope can be inserted through the Chung's needle.

2) Ultra-thin rigid arthroscopy (Fig. 1)

The ultra-thin rigid arthroscopy (EndostarNanoscopy System(Hanseo Medics Co.,Ltd., Seoul, Korea)) for TMJ procedure consists of $167 \mathrm{~mm}$ of total length, $57.5 \mathrm{~mm}$ of available length, a $0.8 \mathrm{~mm}$ diameter fiber optic nanoscope, and a 16 Gauge Needle trocar with irrigation port and a Digital CCD Camera system with S/D card for recording, a 80W LED Light Source, and a 19-inch Kostec Color Video Monitor were used as the monitoring and recording system

\section{Surgical technique}

The patient is prepared and draped in the standard fashion for arthrocentesis. The technique involves a conventional arthroscopic technique through the postero-lateral approach with Chung's needle (16 gauge needle size) under local anesthesia. Approximately 1.0-1.5 mL(pathologic TMJ about less than $1.0 \mathrm{ml}$ ) of lactated Ringer solution is injected into the superior joint space with 


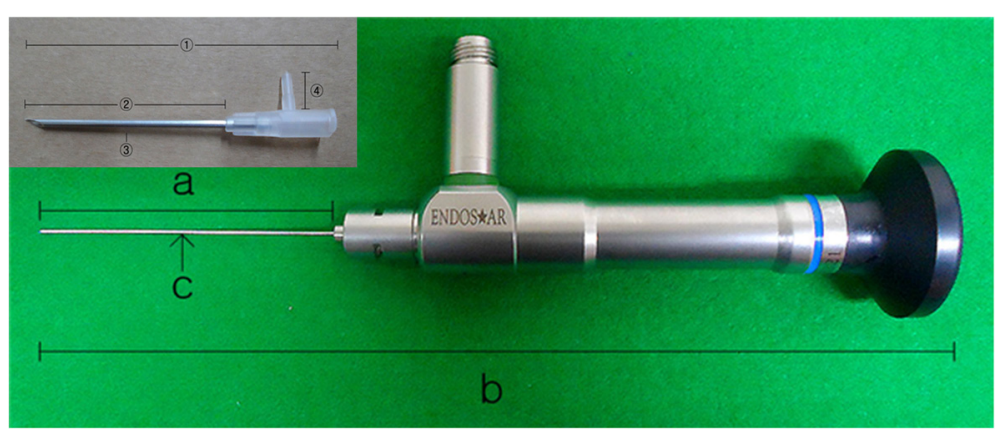

Fig. 1 Chung's needle has $66.20 \mathrm{~mm}$ of total length (1), $43.50 \mathrm{~mm}$ of available length (2), $1.65 \mathrm{~mm}$ of exteranl diameter (3), and $8.30 \mathrm{~mm}$ of irrigation port (4). The ultra-thin rigid arthroscopy (Endostar-Nanoscopy System (Hanseo Medics Co., Ltd., Seoul, Korea)) consists of 167 mm of total length (b), $57.5 \mathrm{~mm}$ of available length (a), a $0.8 \mathrm{~mm}$ diameter fiber optic nanoscope (c)

Chung's needle (16-gauge needle size). After pumping manipulation, closing the needle with 3 way coke for enlarging the superior joint space, and then another $16 \mathrm{G}$ or $18 \mathrm{G}$ needle inserts to the superior joint space for emitting the irrigation solution. With ultra-thin rigid arthroscopy view, joint space has been inspected and arthrocentesis was performed.

For the arthrocentesis, approximately more than $300 \mathrm{ml}$ solution wash out the joint space and then ultra-thin rigid arthroscopy is inserted through the Chung's needle for inspecting the joint space. When encountering the adhesion, resolve with trocar through the Chung's needle and then instruct the mouth opening exercise during irrigation. When encountering the habitual dislocation, injure to the retrodiscal tissue by radiofrequncy (RF) surgery instrument.

After a sufficient range of movement has been achieved, the joint cavity is evaluated with arthroscopy. Finally, sodium hyaluronate (Seikagaku Kogyo Co, Tokyo, Japan) is injected into the superior joint space after joint irrigation, and removed the Chung's needle. A mandibular motion exercise regimen was begun immediately after procedure.

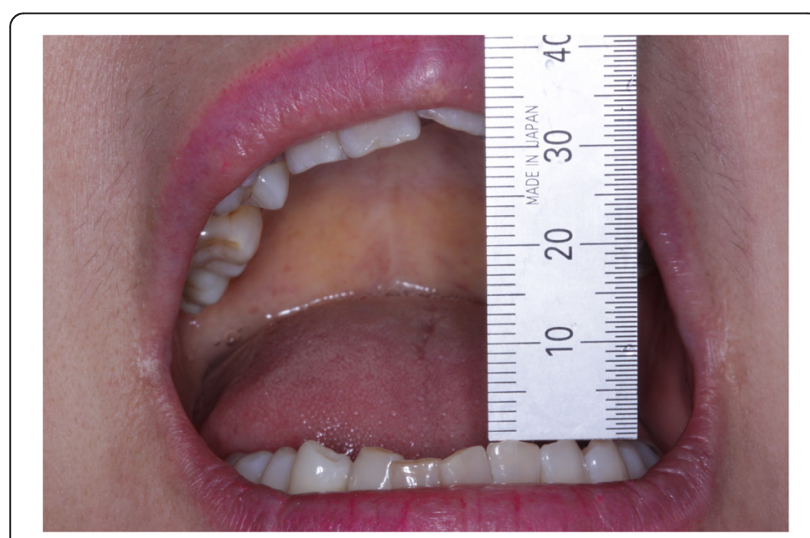

Fig. 223 year-old female was complained of mouth opening limitation, TMJ pain and sound. The mouth opening at initial examination was $32 \mathrm{~mm}$

\section{Case presentation}

\section{Case 1}

Closed lock (anterior disc displacement without reduction)

Twenty three year-old female was complained of mouth opening limitation, TMJ pain and sound. The mouth opening at initial examination was $32 \mathrm{~mm}$ (Fig. 2). The patient has suffered from this problem, and experienced arthrocentesis before. Under diagnosing to chronic closed lock on both TMJ and anterior disk displacement without reduction in MRI (Fig. 3), ultra-thin arthroscopic diagnosis and arthrocentesis was performed, and the adhesion was removed with trocar through the portal of Chung's needle (Figs. 4, 5). After arthrocentesis, range of mouth opening was $45 \mathrm{~mm}$, and symptom was disappeared (Fig. 6).

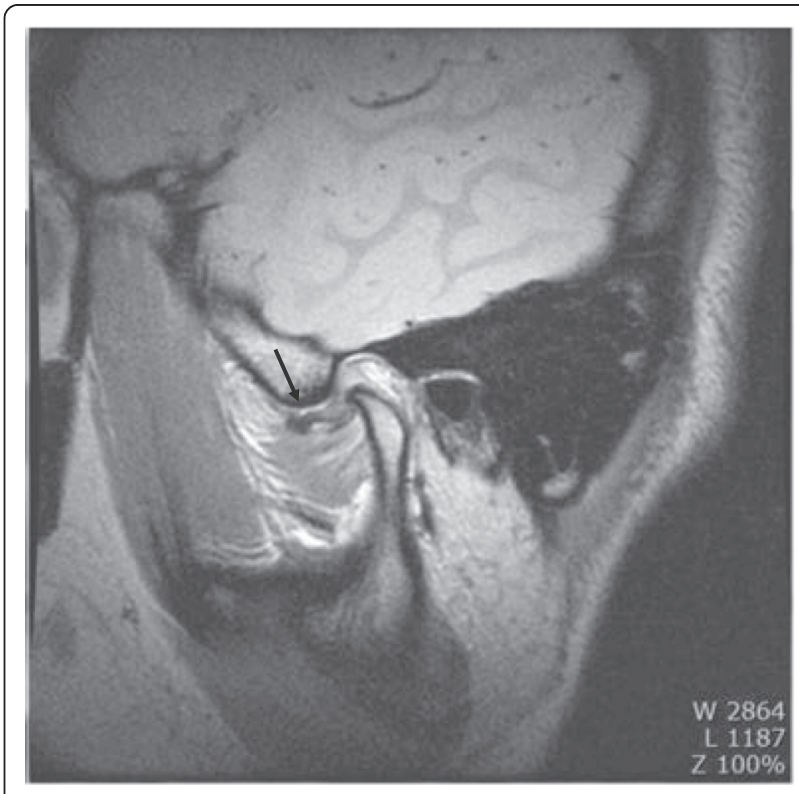

Fig. $3 \mathrm{MRl}$ shows the posterior band of the disc was anterior to the superior part of the condylar head. (Arrow indicates displaced disc anteriorly) 


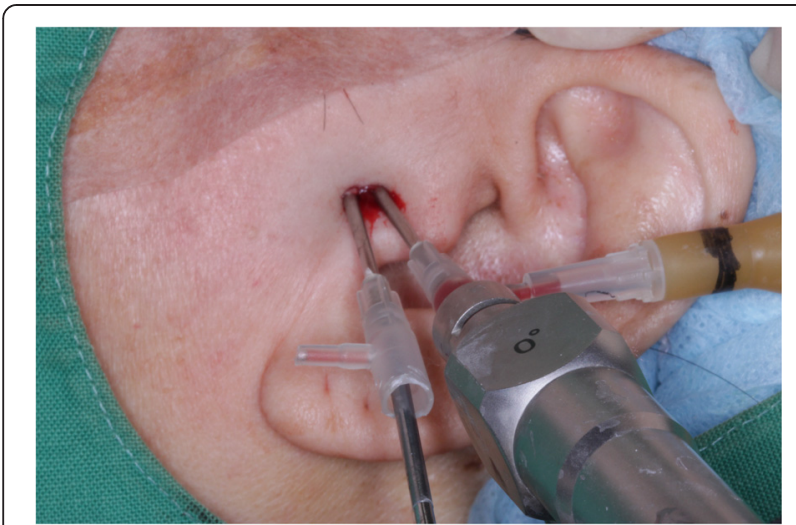

Fig. 4 Ultra-thin arthroscopy inserted through the Chung's needle and trocar was inserted through another portal

\section{Case 2 (Habitual dislocation)}

Twenty two year-old female was complained of habitual dislocation, articular sound and pain. The patient had prior history of TMJ dislocation 3 to 4 times in a week, and reduced by herself. Chung's needle introduced to the superior joint space of the TMJ, inserted the unltrathin arthroscopy and RF surgery tip (Fig. 7) and retrodiscal tissue was injured with RF surgery instrument through the Chung's needle and irrigated the joint space (Fig. 8). The patient had no pain during mouth opening and no episodes of dislocation after treatment.

\section{Discussion}

This study suggests that the ultra-thin rigid arthroscopy showed highly detailed pathologic information of the intra-articular findings with high diagnostic accuracy. Arthrocentesis has been widely used, and several

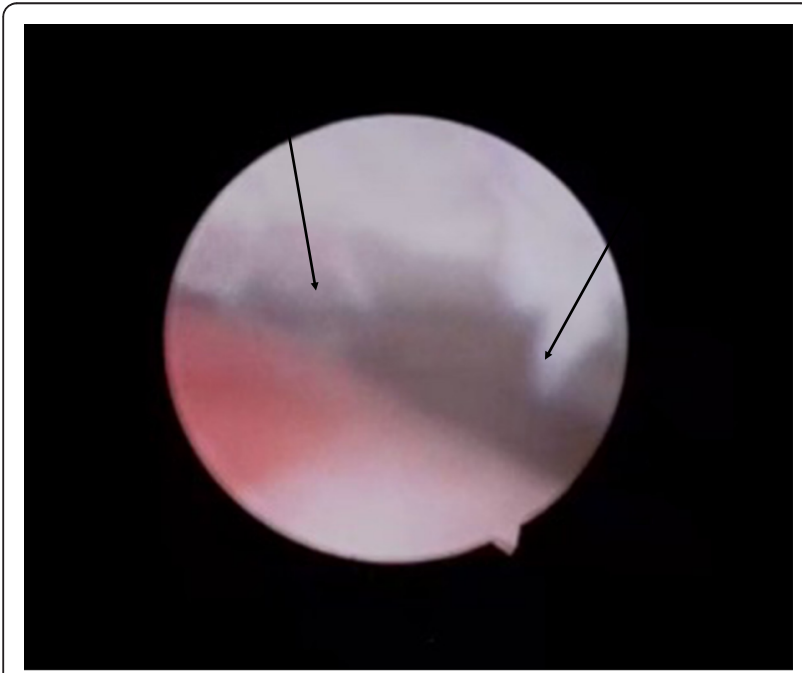

Fig. 5 Adhesion was released with a blunt trocar under arthroscopic view. (Arrow indicates fibrous adhesion)

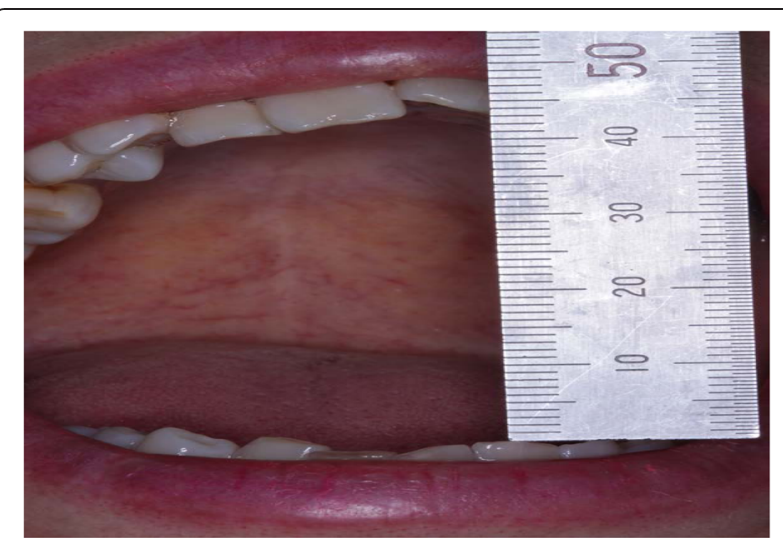

Fig. 6 Range of mouth opening was improved from $32 \mathrm{~mm}$ to $45 \mathrm{~mm}$ on 7 days postoperatively

clinicians have reported on its efficacy and noninvasiveness [7].

The ultra-thin rigid arthroscopy with arthrocentesis has several potential advantages: it provides rapid and easy diagnosis and treatment simultaneously on outpatient basis. Iatrogenic damage is minimal and less bleeding, because of their small size. With this ultra-thin rigid arthroscopy puncture system, it is better to perform the arthrocentesis and diagnose the state of the TMJ than conventional arthroscopy.

Kalunian et al. [8] have described visually guided irrigation (VGIR) of the knee joint. They emphasized that visual guidance during joint irrigation was imperative not only for adequate irrigation of the different joint compartments, but also for the identification of morphologic characteristics that might be important in predicting the outcome. This visual guided irrigation system is similar to our concept in terms of performing arthrocentesis under arthroscopic view. This is a simple and comfortable procedure for the clinician and patient that would make use of a new ultra-thin

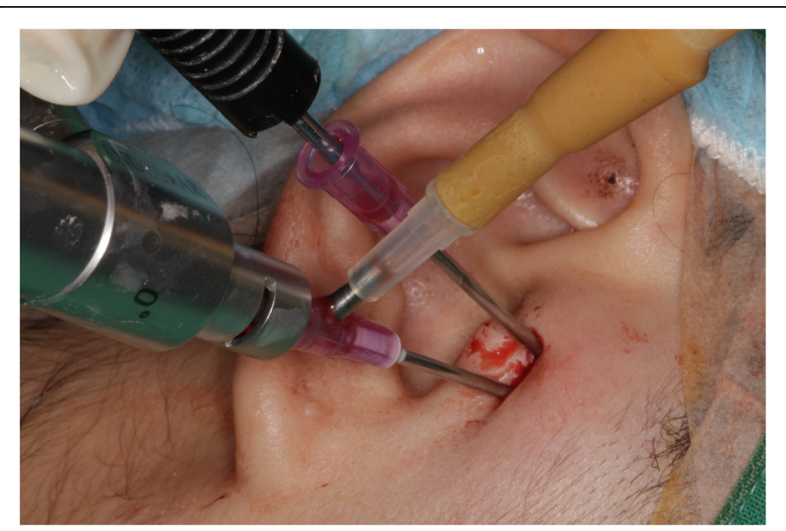

Fig. 7 RF surgery tip inserted through a second portal 


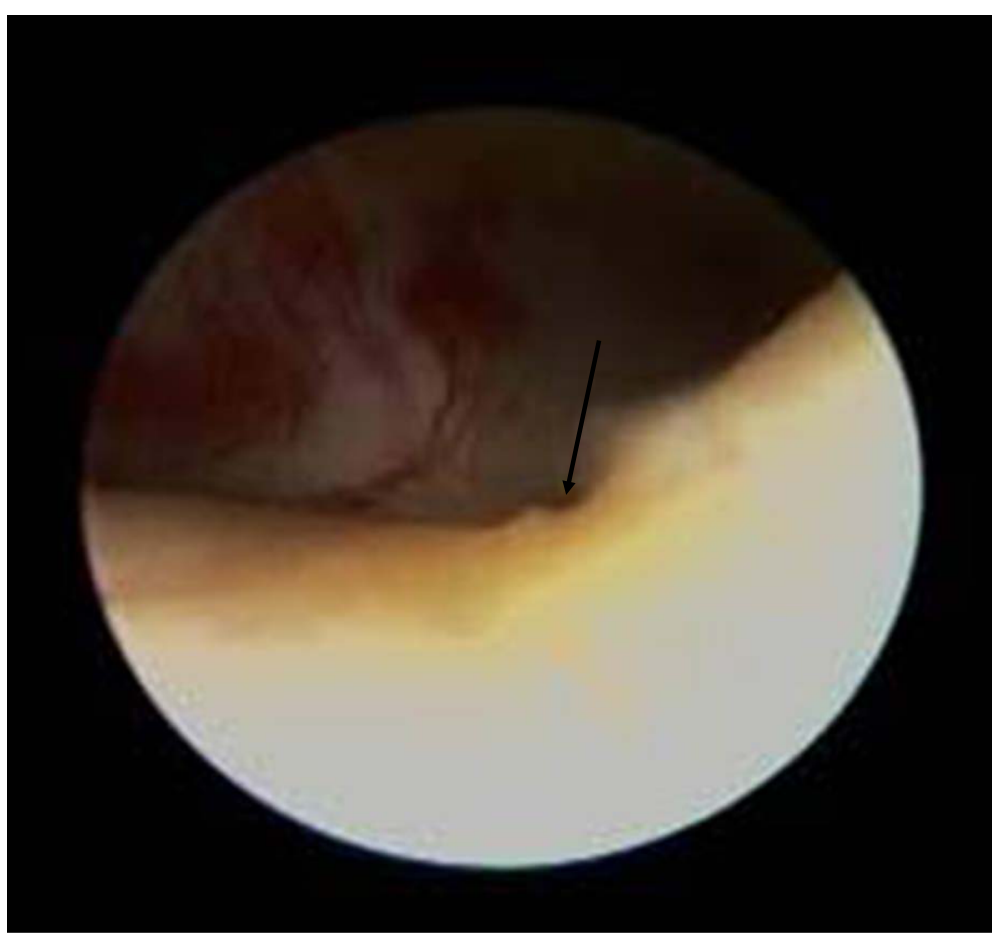

Fig. 8 Retrodiscal tissue was injured with RF surgery instrument under arthroscopic view (Arrow indicates injured retrodiscal tissue)

arthroscopic system with Chung's needle. We thus were able to obtain reliable intra-articular images similar to conventional arthroscope images during combination with irrigation under local anesthesia. This minimally invasive technique also enabled us to compare between clinical outcome parameters and arthroscopic findings. Additionally, it can be used to inspect intra-articular status and treat pathologic conditions in the joint.

\section{Conclusions}

In the present study, we examined morphologic changes in the superior joint space before and after procedure in patients with adhesion and habitual dislocation. Complications were few and patients would be satisfied. This ultra-thin arthroscopy could be used for the management of the TMJ disorders with minimal invasiveness.

\section{Consent}

Written informed consent was obtained from the patient for publication of this Case report and any accompanying images. A copy of the written consent is available for review by the Editor-in-Chief of this journal.

\section{Competing interests}

The authors declare that they have no competing interest.

\section{Authors' contributions}

$\mathrm{HC}$ carried out the arthroscopic surgery, participated in the sequence alignment and drafted the manuscript. S-YM carried out the design of the study and drafted the manuscript. Both authors read and approved the final manuscript.

\section{Acknowledgements}

Seong-Yong Moon was supported by research fund from Chosun University, 2014) in the writing of the manuscript.

\section{Author details}

${ }^{1}$ Department of Oral and Maxillofacial Surgery, School of Dentistry, Chosun University, Gwang-Ju, South Korea. 'Hoon Chung Dental Clinic, \#2305, Jangkyo BLDG.,1 Jangkyo-Dong, Seoul, Jung-Gu Zip Code: 110-760, Republic of Korea.

Received: 4 May 2015 Accepted: 7 July 2015

Published online: 15 July 2015

\section{References}

1. Onishi M (1975) Arthroscopy of the temporomandibular joint (author's transl). Kokubyo Gakkai Zasshi 2:207-213

2. Rao VM, Farole A, Karasick D (1990) Temporomandibular joint dysfunction: correlation of MR imaging, arthrography, and arthroscopy. Radiology 174:663-667

3. Fridrich KL, Wise JM, Zeitler DL (1996) Prospective comparison of arthroscopy and arthrocentesis for temporomandibular joint disorders. J Oral Maxillofac Surg 7:816-820

4. Nitzan DW (2006) Arthrocentesis-incentives for using this minimally invasive approach for temporomandibular disorders. Oral Maxillofac Surg Clin North Am 3:311-328

5. Indresano AT (2001) Surgical arthroscopy as the preferred treatment for internal derangements of the temporomandibular joint. J Oral Maxillofac Surg 3:308-312

6. Kurita K, Ogi N, Miyamoto K, Goss AN (2005) Diagnostic evaluation of an ultrathin 15,000 fiberoptic arthroscope: comparison of arthroscopic and histologic findings in a sheep model. J Oral Maxillofac Surg 3:319-322 
7. Sanders B (1986) Arthroscopic surgery of the temporomandibular joint: treatment of internal derangement with persistent closed lock. Oral Surg Oral Med Oral Pathol 4:361-372

8. Kalunian KC, Moreland LW, Klashman DJ, Brion PH, Concoff AL, Myers S, Singh R, Ike RW, Seeger LL, Rich E, Skovron ML (2000) Visually-guided irrigation in patients with early knee osteoarthritis: a multicenter randomized, controlled trial. Osteoarthritis Cartilage 6:412-418

Submit your manuscript to a SpringerOpen ${ }^{\odot}$ journal and benefit from:

- Convenient online submission

Rigorous peer review

- Immediate publication on acceptance

- Open access: articles freely available online

- High visibility within the field

- Retaining the copyright to your article

Submit your next manuscript at $\gg$ springeropen.com 